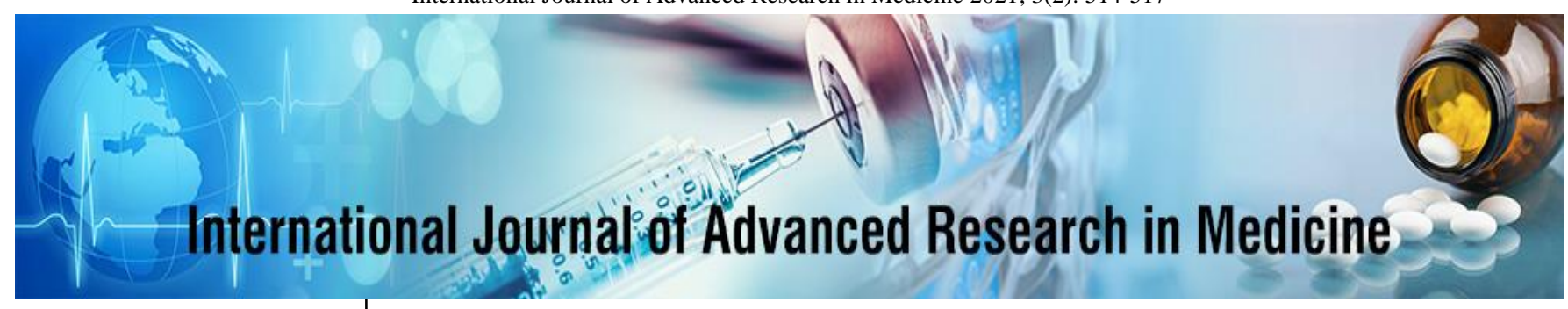

E-ISSN: 2706-9575

P-ISSN: 2706-9567

IJARM 2021; 3(2): 514-517

Received: 03-06-2021

Accepted: 05-08-2021

Dr. Venkappa S Mantur

Assistant Professor,

Department of Physiology,

SSPM Medical College and Life

Time Hospital, Padve,

Sindhudurg, Maharashtra,

India

Dr. Sudarshan Reddy

Associate Professor,

Department of Pharmacology,

SSPM Medical College and Life

Time Hospital, Padve,

Sindhudurg, Maharashtra,

India

Dr. Arun Kumar

Associate Professor,

Department of Biochemistry,

SSPM Medical College and Life

Time Hospital, Padve,

Sindhudurg, Maharashtra,

India

Corresponding Author: Dr. Venkappa S Mantur Assistant Professor, Department of Physiology, SSPM Medical College and Life Time Hospital, Padve, Sindhudurg, Maharashtra, India

\section{Do the stress levels affect the quality of life and treatment adherence in hospitalized medically ill patients?}

\author{
Dr. Venkappa S Mantur, Dr. Sudarshan Reddy and Dr. Arun Kumar
}

DOI: https://doi.org/10.22271/27069567.2021.v3.i2h.300

\begin{abstract}
Background and Objectives: Stress can be experienced in day to day life and can be usually resolved by effective coping mechanisms of a person and can also prove beneficial provided the loss of equilibrium is corrected. However, some life events may disturb the steady state to an extent where the person is not able to overcome the stress. In this study, we aimed to examine the relationship between perceived stress levels, quality of life and treatment adherence in patients hospitalized with medical illnesses. 400 patients admitted in the general medicine ward were approached and, after obtaining informed consent, perceived stress, quality of life and treatment adherence was assessed using the Perceived Stress Scale (PSS-10), World Health Organization Quality of Life scale-Bref version and Morisky Medication Adherence Questionnaire. Socio demographic and clinical features were also assessed and relationship between all the variables was studied.
\end{abstract}

Keywords: perceived stress, quality of life, medically ill

\section{Introduction}

Stress can be experienced in day to day life and can be usually resolved by effective coping mechanisms of a person and can also prove beneficial provided the loss of equilibrium is corrected. However, some life events may disturb the steady state to an extent where the person is not able to overcome the stress.

Stress is "any external event or internal drive which threatens to upset the organismic equilibrium" "[1]. Stress has also been defined as a state of imbalance with in an organism that is elicited by an actual/perceived disparity between environmental demands and the organism's capacity to cope with these demands; and is manifested through a variety of physiological, emotional and behavioural responses ${ }^{[2]}$.

Medical illness is one such state where, at times, the person may find it difficult to overcome the stress. Medical illnesses and stress have a bi-directional relationship. Medical illnesses usually lead to an increase in perceived stress of the person and the stress will, in turn, adversely affect the outcome of the illness in some conditions. Hospitalization can lead to a further increase in stress as it can be a very traumatic experience where people have to move their life setting from a familiar to an unfamiliar environment, with the loss of a sense of security and individuality ${ }^{[3]}$.

Quality of life has been defined as “an individuals' perceptions of their position in life in the context of the culture and value systems in which they live, and in relation to their goals, expectations, standards, concerns". It is a broad ranging concept affected in a complex way by the persons' physical health, psychological state, level of independence, social relationships and their relationship to salient features of their environment ${ }^{[4]}$. Quality of life has been shown to be negatively affected in patients suffering from illnesses like diabetes mellitus, hypertension and tuberculosis and often found to be associated with higher levels of stress and psychological co-morbidities ${ }^{[5,6]}$. Quality of life has also been linked to treatment adherence with poor treatment adherence often associated with poor quality of life ${ }^{[7]}$.

\section{Objectives}

To study the levels of perceived stress and the quality of life and their association in medically ill hospitalized patients. 


\section{Materials and Methods}

- Design: Cross-sectional design.

- Study population and setting: 400 patients admitted in the general medicine wards.

- 400 patients admitted in the general medicine ward, who met the inclusion criteria and gave written informed consent, were assessed using the Perceived Stress Scale, World Health Organization Quality of Life scale-Bref version and Morisky Medication Adherence Questionnaire. Socio demographic and clinical features were also assessed and the relationship between all the aforementioned variables was studied.

\section{Results}

Table 1: Perceived Stress Scale, Quality of Life and Treatment Adherence scores

\begin{tabular}{|c|c|}
\hline \multicolumn{2}{|c|}{ Perceived Stress Scale } \\
\hline \multicolumn{2}{|c|}{ Mean (SD) } \\
\hline Total score & $16.17(5.19)$ \\
\hline WHOQOL-BREF \\
\hline Overall quality of life and general health & $7.37(1.62)$ \\
\hline Physical health & $25.57(4.39)$ \\
\hline Psychological health & $22.33(4.17)$ \\
\hline Social relationships & $11.68(1.68)$ \\
\hline Environment & $28.92(4.3)$ \\
\hline MMAS-4 \\
\hline Total score & $2.11(1.41)$ \\
\hline
\end{tabular}

Perceived stress, quality of life and treatment adherence were assessed using the PSS-10, WHOQOL-BREF and MMAS-4 respectively

Table 2: Relationship between perceived stress and quality of life

\begin{tabular}{|c|c|}
\hline Variable & $\begin{array}{c}\text { Correlation coefficient } \\
\text { value) }\end{array}$ \\
\hline $\begin{array}{c}\text { Overall quality of life and general } \\
\text { health }\end{array}$ & $(<0.001)$ \\
\hline Physical health & $(<0.001)$ \\
\hline Psychological health & $(<0.001)$ \\
\hline Social relationships & $(<0.001)$ \\
\hline Environment & $(<0.001)$ \\
\hline PSS total score & ------------- \\
\hline
\end{tabular}

Table 3: Regression analysis showing the ability of perceived stress to predict the quality of life and treatment adherence (enter method) $[\mathrm{N}=400]$

\begin{tabular}{|c|c|c|}
\hline Dependent Variables & $\begin{array}{c}\text { Adjusted R } \\
\text { Square }\end{array}$ & $\begin{array}{c}\text { td. Error of the } \\
\text { Estimate }\end{array}$ \\
\hline \multicolumn{2}{|c|}{ WHOQOL-BREF Overall quality of life and general health } \\
\hline Perceived stress score (predictor) & .313 & 1.39 \\
\hline \multicolumn{2}{|c|}{ WHOQOLBREF-Physical domain } \\
\hline Perceived stress score (predictor) & .374 & 3.41 \\
\hline \multicolumn{2}{|c|}{ WHOQOLBREF-Psychological domain } \\
\hline Perceived stress score (predictor) & .469 & 2.83 \\
\hline \multicolumn{2}{|c|}{ WHOQOLBREF-Social domain } \\
\hline Perceived stress score (predictor) & .143 & 1.59 \\
\hline \multicolumn{2}{|c|}{ WHOQOLBREF-Environment domain } \\
\hline Perceived stress score (predictor) & .269 & 3.28 \\
\hline
\end{tabular}

Regression analysis was run to explain the extent to which the perceived stress explained the variance in quality of life and the treatment adherence. As seen in table, perceived stress explained to the variance of different domains of quality of life as per WHOQOL-BREF, with the social relationships domain being the least and psychological health being the highest.

\section{Discussion}

Numerous studies have been conducted, in India and abroad, to assess the levels of stress, the quality of life and treatment adherence in patients suffering from various medical illnesses. Some of the studies have also tried to understand the association between the aforementioned factors. While some of the research focuses on the stress and quality of life in hospitalized patients, the rest of the studies targeted patients staying in the community and attending the hospitals as out-patients.

\section{Prevalence rates of stress, quality of life and treatment adherence}

Medical illnesses often lead to an increase in the stress levels of the patients due to various factors like the distress caused by the symptoms, restriction of movement and functioning, financial costs of treatment and worries about the prognosis and the future. Several studies have attempted to assess the level of stress in patients with different medical conditions and its impact on the symptoms and the prognosis.

In a study involving 181 patients suffering from Chronic Obstructive Lung Disease (COPD), mean perceived stress score assessed using the 14-item Perceived Stress Scale (PSS-14) was found to be 22.8, indicating moderate amount of stress. More shortness of breath and greater functional impairment were reported by those with higher stress scores [8]. In another study comparing the perceived stress levels between women and men immediately and 12 months after acute myocardial infarction, both groups were found to have moderate levels of stress as assessed by the PSS-14. Perceived stress scores were found to decline over the twelve months period and women reported higher perceived stress compared to men both immediately and twelve months after myocardial infarction ${ }^{[9]}$.

Stress levels are often especially high in patients who suffer from chronic diseases with severe impairment of functioning. A study of 80 patients with chronic kidney disease undergoing dialysis in dialysis centres in South India revealed significantly high levels of stress ${ }^{[10]}$. In patients with chronic kidney disease undergoing dialysis, it has been noted that financial difficulties, change in marital and social relationships, fear of disability and death and uncertainty about the future may worsen the stress and may also lead to comorbid mental health problems like depression and anxiety ${ }^{[11]}$

Hypertension is one of the most common chronic diseases to be often linked with stress. A transient elevation in blood pressure is often noted in stressful conditions. Some studies have attempted to study the association between stress and hypertension. Two studies done in India have reported significantly high levels of stress in patients with hypertension. While one study reported the prevalence of stress to be $84.3 \%$, the other study reported $91.2 \%$ of the hypertensive patients to be having high level of stress ${ }^{[12,13]}$. A study analysed the data from the World health Survey (2002-2004), including 229,293 adults, and assessed the association of chronic medical conditions and multi morbidity with perceived stress among community-dwelling adults in 44 low and middle-income countries. Perceived stress was assessed along with eleven chronic conditions 
(angina, arthritis, asthma, chronic back pain, depression, and diabetes, edentulism, hearing problem, schizophrenia, tuberculosis and visual impairment). With the exception of edentulism, all the other chronic conditions were associated with higher mean perceived stress scores and the poorest individuals were deemed to be particularly vulnerable with regards to higher perceived stress ${ }^{[14]}$.

In addition to the prevailing medical illnesses, hospitalization further adds to the stress level due to movement to an unfamiliar environment and the financial burden of hospitalization accompanied by inability to earn income while admitted. In a study done in 700 hospitalized patients across various departments in central India, $72.9 \%$ of the patients reported stress post hospitalization, with women and patients hailing from urban areas reporting higher levels of stress. Some of the major factors for stress were found to be possible loss of body part or function and uncertainty regarding outcome of treatment ${ }^{[3]}$.

In a study on 80 hospitalized patients with COPD in Spain, stress was assessed on Hospital Stress rating scale (HSRS) and the stress score (166.4) was found to be lower than the mean score of the HSRS scale (220.5). The items related to the change of habits, loss of control, loss of autonomy and privacy were considered by the COPD patients to be the most stressful items during hospitalization ${ }^{[15]}$.

Quality of life (QOL) is an important multi-dimensional construct which is considered vital in assessing the burden of the disease and the impact on the functioning of an individual. Chronic diseases may impair the quality of life of the patients by limiting their capacity to interact and function in the day to day life, to earn a living and due to mounting medical costs ${ }^{[16]}$. A vast amount of research has been conducted assessing the quality of life and its correlates in individuals suffering from various medical disorders.

A study from India assessing quality of life in 90 patients with tuberculosis found lower quality of life scores in all four domains of the WHOQOL-BREF compared to the control group ${ }^{[5]}$. Similarly, in a health survey done in China in 1,224 adults, hypertension was found to be associated with a poorer health related quality of life with lower scores of physical functioning, role-physical, bodily pain, general health, vitality, and social function in individuals with hypertension compared to those without hypertension ${ }^{[17]}$.

In a study done on 200 patients with diabetes attending a rural primary care centre in Kerala, it was found that the percentage of people occupying more than 75th percentile in the WHOQOL-BREF domain scores corresponded to $7.1 \%$, $16.2 \%, 17.25 \%$, and $40.1 \%$ in the physical, psychological, social and environmental domains respectively, indicating an overall poor QOL in all domains ${ }^{[18]}$.

Similarly, in a cross-sectional study done on 75 patients undergoing haemodialysis for at least 3 months, significant impairment in quality of life scores in the physical, psychological and social domains of WHOQOL-BREF was noted ${ }^{[19]}$.

Treatment adherence, or medication adherence, has been defined by the World Health Organization as "the extent to which a person's behaviour - taking medication, following a diet, and/or executing lifestyle changes - corresponds with agreed recommendations from a health care provider". Nonadherence to treatment is often observed due to various factors ranging from personal beliefs of the patients and the caregivers like misconceptions regarding the medications, adverse effects of the medications, difficulty in bearing the treatment costs and comorbid conditions like depression leading to a sense of hopelessness regarding the illness and treatment. Non-adherence rates for the numerous chronic conditions vary from $23 \%$ to nearly $75 \%$ in the several studies ${ }^{[20-23]}$.

Treatment adherence can be examined either by use of subjective questionnaires like Morisky medication adherence scale ( 4 and 8 item) or by counting of pills and examining the pharmacy refill record ${ }^{[24]}$. Most of the studies have used different questionnaires to examine the rates of treatment adherence.

In hypertensive patients on anti-hypertensive treatment, treatment non-adherence rates have been found to vary from $20 \%$ to nearly $75 \%$. Some of the factors associated with poor treatment adherence were poor awareness regarding the illness, less educated, adverse effects with medicines and hailing from rural areas with poor access to healthcare facilities $[20,21,25,26]$.

In a study done on 133 COPD patients, treatment nonadherence was found to be $45 \%$, as measured by the Morisky medication adherence scale, with the duration of illness being a risk factor for treatment non-adherence ${ }^{[2]}$.

A study on 150 patients with chronic kidney disease revealed non-adherence rates of $34 \%$ with high costs, complex dosing schedule and fear of adverse effects being the prominent reasons for the same ${ }^{[23]}$.

\section{Conclusions and implications}

Medical illness and hospitalization are stressful situations which significantly impair the quality of life. Majority of the patients show significant amounts of perceived stress. Most patients reported a high quality of life in terms of physical health, psychological health, social relationships and environment. Partial treatment adherence was seen in most patients. Perceived stress is significantly associated with a lower quality of life in all domains and lower levels of treatment adherence. Better treatment adherence is associated with greater quality of life in physical health, psychological health and environment domains.

\section{References}

1. Selye H. The stress of life. New York: McGraw-Hill, 1956.

2. Lazarus RS. Psychological stress and the coping process. New York: McGraw-Hill, 1966.

3. Chhari N, Mehta SC. Stress among Patients during Hospitalization: A Study from Central India. Natl J Community Med 2016;7:274-7.

4. WHOQOL Group. The World Health Organization quality of life assessment (WHOQOL): position paper from the World Health Organization. Soc Sci Med 1995;41:1403-9.

5. Dhuria M, Sharma N, Ingle GK. Impact of tuberculosis on the quality of life. Indian $\mathbf{J}$ Community Med 2008;33:58-9.

6. Testa MA, Simonson DC. Measuring quality of life in hypertensive patients with diabetes. Postgrad Med J 1988;64:50-8.

7. Alhaddad IA, Hamoui O, Hammoudeh A, Mallat S. Treatment adherence and quality of life in patients on antihypertensive medications in a Middle Eastern population: adherence. Vasc Health Risk Manag 2016;12:407-13. 
8. Delgado C. Sense of coherence, spirituality, stress and quality of life in chronic illness. J Nurs Scholarsh 2007;39:229-34.

9. $\mathrm{Xu} \mathrm{X}, \mathrm{Bao} \mathrm{H}$, Strait KM, Edmondson DE, Davidson KW, Beltrame JF, et al. Perceived stress after acute myocardial infarction: A comparison between young and middle-aged women versus men. Psychosom Med 2017;79:50-8.

10. Jose K, Saji S, Thomas A. Influence of demographic factors on perceived stress and quality of life in persons with chronic kidney disease: a study from South India. Indian J Appl Res 2019;9:7-9.

11. Gerogianni SK, Babatsikou FP. Psychological aspects in chronic renal failure. Health Sci J 2014;8:205-14.

12. Sarkar A, Roy D, Chauhan MM, Dave P, Makwana NR, Parmar DV. A lay epidemiological study on coexistent stress in hypertension: Its prevalence, risk factors, and implications in patients' lives. J Family Med Prim Care 2019;8:966-71.

13. Bhelkar S, Deshpande S, Mankar S, Hiwarkar P. Association Between Stress And Hypertension Among Adults More Than 30 Years: A Case-Control Study. National J Comm Med 2018;9:430-3.

14. Vancampfort D, Koyanagi A, Ward PB, Veronese N, Carvalho AF, Solmi M, et al. Perceived stress and its relationship with chronic medical conditions and multi morbidity among 229,293 community-dwelling adults in 44 low-and middle-income countries. Am J Epidemiol 2017;186:979-89.

15. Medinas-Amorós M, Montaño-Moreno JJ, CentenoFlores MJ, Ferrer-Pérez V, Renom-Sotorra F, MartínLópez B, et al. Stress associated with hospitalization in patients with COPD: the role of social support and health related quality of life. Multidiscip Respir Med 2012;7:51.

16. Megari K. Quality of life in chronic disease patients. Health Psychol Res 2013;1:e27.

17. Xu X, Rao Y, Shi Z, Liu L, Chen C, Zhao Y. Hypertension impact on health-related quality of life: a cross-sectional survey among middle-aged adults in Chongqing, China. Int J Hypertens 2016;2016:7404957.

18. Sreedevi A, Cherkil S, Kuttikattu DS, Kamalamma L, Oldenburg B. Validation of WHOQOL-BREF in Malayalam and determinants of quality of life among people with type 2 diabetes in Kerala, India. Asia Pac J Public Health 2016;28:62S-9S.

19. Sathvik BS, Parthasarathi G, Narahari MG, Gurudev KC. An assessment of the quality of life in hemodialysis patients using the WHOQOL-BREF questionnaire. Indian J Nephrol 2008;18:141-9.

20. Balasubramanian A, Nair SS, Rakesh PS, Leelamoni K. Adherence to treatment among hypertensives of rural Kerala, India. J Family Med Prim Care 2018;7:64-9.

21. Gupta M, Bhattacharjee A, Singh H. Evaluation of medication adherence patterns amongst antihypertensive drug users in a tertiary care hospital in north India. Int J Basic Clin Pharmacol. 2017;5:2261-6.

22. Mohsen S, Hanafy FZ, Fathy AA, El-Gilany AH. Nonadherence to treatment and quality of life among patients with chronic obstructive pulmonary disease. Lung India 2019;36:193-8.

23. Sontakke S, Budania R, Bajait C, Jaiswal K, Pimpalkhute S. Evaluation of adherence to therapy in patients of chronic kidney disease. Indian J Pharmacol 2015;47:668-71.

24. Singh P. Medication adherence: Adjunct to patient outcomes. J Pract Cardiovasc Sci 2017;3:8-10.

25. Hashmi SK, Afridi MB, Abbas K, Sajwani RA, Saleheen D, Frossard PM, et al. Factors associated with adherence to anti-hypertensive treatment in Pakistan. PLoS One 2007;2:e280.

26. Al-Ramahi R. Adherence to medications and associated factors: A cross-sectional study among Palestinian hypertensive patients. J Epidemiol Glob Health 2015;5:125-32. 